\title{
An unusual presentation of Behçet's disease
}

\author{
W K M G Amarawardena, A Wijesundere, W M T Muhandiram, H S D Appuhamy
}

Ceylon Medical Journal 2014; 59: 144-145

\section{Introduction}

Behçet's disease (BD) is a chronic relapsing systemic vasculitis that can involve almost every organ and system in the body with diverse manifestations. Both arteries and veins may be involved. Venous complications are commoner and range from superficial thromboplebitis to deep vein thrombosis [1,2]. However, chylothorax due to superior vena cava (SVC) obstruction is a rare complication of $\mathrm{BD}[3]$.

\section{Case report}

A 38-years old single lady presented with shortness of breath and facial oedema, but no significant ankle oedema or abdominal distension. She also complained of recurrent painful genital ulcers and oral ulcers of one year duration. She developed pustular lesions over the face recently. She denied sexual promiscuity, genital discharge or chronic diarrohea. On examination she had facial puffiness with distended veins over the upper trunk and a palpable mass over the right side of neck. Jugular venous pressure was normal. There was evidence of pleural effusion on the right side.

A clinical diagnosis of SVC obstruction was made and ultrasound scan of the neck was performed. This showed a thrombus in the cervical part of the left internal jugular vein. Contrast CT neck and chest showed non enhancing intraluminal low attenuation in the left subclavian vein, left brachiocephalic vein and SVC, suggestive of venous thrombosis. There was mild to moderate right sided pleural effusion with low attenuation material (HU value -11 to +5 ) which suggested a chylothorax. The conclusion was superior vena cava syndrome with evidence of opening up of multiple collateral venous channels.

Pleural fluid report showed glucose $130.3 \mathrm{mg} / \mathrm{dl}$, proteins $50.2 \mathrm{~g} / \mathrm{dl}$, LDH $9.5 \mathrm{iu} / 1$, cholesterol $96.4 \mathrm{mg} /$ $\mathrm{dl}$, triglycerides $358 \mathrm{mg} / \mathrm{dl}, \mathrm{pH}=7.5$, leucocytes 12160 / $\mathrm{mm}^{3}$, with $90 \%$ lymphocytes, TB PCR was negative. Inflammatory markers were elevated ESR $98 \mathrm{~mm} / 1^{\text {st }}$ hour, CRP $108 \mathrm{mu} / \mathrm{dl}$. Antinuclear antibody, double stranded DNA, anti-cardiolipin antibodies, HSV serology, VDRL and repeated HIV antibody tests were negative. Pathergy test was positive. A clinical diagnosis of BD was made. She was commenced on prednisolone $30 \mathrm{mg}$ per day

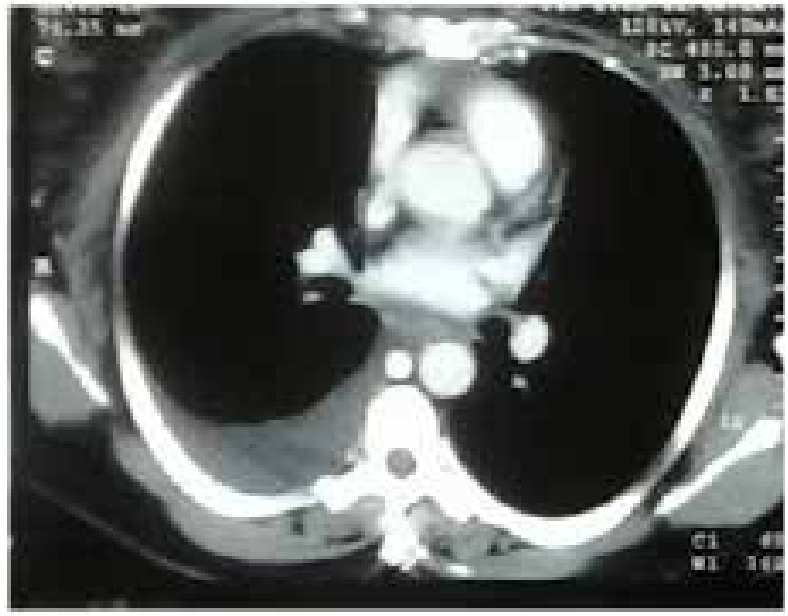

Figure 1. Contrast $\mathrm{CT}$ showing superior vena cava obstruction with chylothorax.

and anti coagulation with low molecular weight heparin and warfarin. With treatment, the inflammatory markers became normal and chylothorax completely resolved.

She traveled overseas for employment and defaulted treatment despite proper education regarding her disease. Six months later, she came with shortness of breath and was found to have a large pleural effusion. Chylothorax was reconfirmed and an inter-costal tube was inserted. As the chylothorax continued to drain for more than two weeks, cardiothoracic opinion was obtained. Thoracic duct was ligated at the level just below the right inferior pulmonary vein at the lower one third of the oesophagus. She recovered from the chylothorax completely. She continued warfarin and prednisolone. After six months she was free of symptoms.

\section{Discussion}

Behçet's disease is a disease known since the time of Hippocrates. It was first defined as a distinct clinical syndrome by a Turkish dermatologist, Dr. Hulusi Behçet, who described three patients with the classic triad of signs. This triad consisted of oral ulcers, genital ulcers, and irido-cyclitis with hypopyon [4]. Studies have demonstrated that this disease is not restricted to these three areas but may also involve the musculoskeletal,

Medical Unit, Sri Jayewardenepura General Hospital, Sri Lanka.

Correspondence: WKMGA, e-mail: <maheshiamarawardena@gmail.com>. Received 8 April 2014 and revised version accepted 6 October 2014. Competing interests: none declared. 
pulmonary, gastrointestinal, urogenital, cardiac, vascular, and neurological systems [4].

In our patient recurrent oral ulcers, genital ulcers, pustular lesions over the face together with a positive pathergy test confirmed Behçet's disease according to the diagnostic criteria proposed by the International Study Group for Behçet's disease [5]. Retroviral infection, herpes simplex infection, and tuberculosis were excluded by relevant investigations. The $\mathrm{CT}$ scan confirmed superior vena cava obstruction and chylothorax was confirmed by elevated triglycerides in the lymphocytic exudative pleural effusion.

The pathogenesis of chylous effusion in BD is uncertain. It has been hypothesised that thrombosis of the subclavian vein may lead to obstruction of the orifice of the thoracic duct. As a result, intraluminal pressure increases and chyle leaks out from the pleural lymphatics into the pleural space. Some authors have proposed two other mechanisms that may result in chylous effusion, both are yet to be confirmed. Firstly, lym-phangitis of the thoracic duct, akin to the vasculitis of $\mathrm{BD}$, could lead to chylous effusion. Secondly, amyloidosis complicating BD could intrinsically obstruct the lymphatics $[6,7]$.

Immunosuppressants are important in the treatment of BD. After treatment with steroids the patient's inflammatory markers returned to normal. Anticoagulation with heparin and warfarin is standard treatment for deep vein thrombosis. It is difficult to treat chylothorax secondary to obstruction of the SVC, and this often requires surgical intervention.

\section{Acknowledgements}

We acknowledge Dr. DMS Handagala, Consultant Cardio-thoracic Surgeon, Chest Hospital, Welisara and the staff, Departement of Radiology, Sri Jayewardenepura General Hospital.

\section{References}

1. Calamia KT, Schirmer M, Melikoglu M. Major vessel involvement in Behcet's. Current Opinion in Rheumatology Review 2005; 17: 1-8.

2. Sakane T, Takeno M, Suzuki N, Inaba G. Behcet's disease. New England Journal of Medicine 1999; 341: 1284-91.

3. Roguin A, Edelstein S, Edoute Y. Superior vena cava syndrome as a primary manifestation of Behçet's disease. A case report. Journal of Angiology 1997; 48: 365-8.

4. Onder M, Gurer MA. The multiple faces of Behçet's disease and its etiological factors. Journalof European Academy of Dermatology and Venereology 2001; 15: 126-36.

5. International Study Group for Behçet's Disease. Criteria for diagnosis of Behçet's disease. Lancet 1990; 335: 107880 .

6. Moon H, Lee YJ, Lee SI, Yoo WH. Chylothorax and chylopericardium as the initial clinical manifestation of Behcet's disease. Rheumatology International 2008; 28: 375-7.

7. Coplu L, Emri S, Selcuk ZT, et al. Life threatening chylouspleural and pericardial effusion in a patient with Behçet's syndrome. Thorax 1992; 47: 64-5. 